\title{
PCM1-JAK2 Fusion in a Patient With Acute Myeloid Leukemia
}

\author{
Jong-Mi Lee, M.D. ${ }^{1,2}$, Jaewoong Lee, M.D. ${ }^{1,2}$, Eunhee Han, M.D. ${ }^{1,2}$, Myungshin Kim ${ }^{\oplus}$, M.D. ${ }^{1,2}$, Yonggoo Kim, M.D. ${ }^{1,2}$,
} Kyungja Han, M.D. ${ }^{1,2}$, and Hee-Je Kim, M.D. ${ }^{3}$

Department of Laboratory Medicine ${ }^{1}$ and Catholic Genetic Laboratory Center ${ }^{2}$, Seoul St. Mary's Hospital, College of Medicine, The Catholic University of Korea, Seoul; Department of Hematology ${ }^{3}$, Catholic Blood and Marrow Transplantation Center, Seoul St. Mary's Hospital, Leukemia Research Institute, College of Medicine, The Catholic University of Korea, Seoul, Korea

Dear Editor,

Over 34 myeloid neoplasm cases with a PCM1-JAK2 fusion have been reported worldwide since 1999, including one case reported in Korea [1, 2]. These cases share common features, such as splenomegaly, eosinophilia, myelofibrosis, and male predominance. Most cases have been diagnosed as myeloproliferative neoplasm or myelodysplastic/myeloproliferative neoplasm, in particular, as chronic eosinophilic leukemia and atypical chronic myeloid leukemia. However, acute myeloid/lymphoid leukemia cases have also been reported [1]. Therefore, the 2016 WHO revision recognized the PCM1-JAK2 fusion gene as a provisional entity [3], joining the existing category of "myeloid and lymphoid neoplasms with eosinophilia and abnormalities of platelet-derived growth factor receptor $\alpha$ (PDGFRA), platelet-derived growth factor receptor $\beta$ (PDGFRB), or fibroblast growth factor receptor 1 (FGFR1)."

A PCM1-JAK2 diagnosis can be made with or without eosinophilia, if the presence of the genetic rearrangement is proven. However, identification of the genomic breakpoint in the PCM1JAK2 fusion is quite difficult because it varies by case; as many as 14 different fusion transcripts from 15 patients have been reported [2, 4-6]. Moreover, the genetic lesions involved in the re- arrangement of the two genes are widely distributed, from exon 23 to exon 36 in PCM1 and from exon 1 to exon 11 in JAK2 [5]. Therefore, it is important to report cases of newly confirmed breakage sites where chromosomal assays offer the only indication of a PCM1-JAK2 fusion. We report a case of acute myeloid leukemia with a PCM1-JAK2 fusion that was not accompanied by eosinophilia. This study was approved by the institutional review board of Seoul St. Mary's Hospital, Korea, and informed consent was obtained from the patient. A 51-year-old woman was diagnosed as having acute myeloid leukemia at a tertiary hospital in September 2009. At diagnosis, a complete blood count revealed $1.8 \times 10^{9} / \mathrm{L}$ leukocytes, $66 \mathrm{~g} / \mathrm{L} \mathrm{Hb}$, and $34 \times 10^{9} / \mathrm{L}$ platelets. A peripheral blood smear demonstrated a leukoerythroblastic reaction (nucleated red blood cells: 86/100 leukocytes; left shifted maturation pattern). A bone marrow (BM) biopsy showed hypercellularity with increased erythroid precursors (64\% of all nucleated cells) and blasts (47\% of non-erythroid cells). The blasts were positive for CD13, CD33, CD117, human leukocyte antigen-antigen D related (HLA-DR), and myeloperoxidase (MPO), and were weakly positive for CD64, CD56, and nuclear terminal deoxynucleotidyl transferase (TdT). A diagnosis of acute erythroid leukemia was made based on the 2008 WHO
Received: August 31, 2017

Revision received: December 17, 2017

Accepted: April 26, 2018

\section{Corresponding author: Myungshin Kim}

(D) https://orcid.org/0000-0001-8632-0168

Department of Laboratory Medicine, College of Medicine, Seoul St. Mary's

Hospital, The Catholic University of Korea, 222 Banpo-daero, Seocho-gu,

Seoul 06591, Korea

Tel: +82-2-2258-1645, Fax: +82-2-2258-1719

E-mail: microkim@catholic.ac.kr

\section{() Korean Society for Laboratory Medicine}

This is an Open Access article distributed under the terms of the Creative Commons Attribution Non-Commercial License (http://creativecommons.org/licenses/by-nc/4.0) which permits unrestricted non-commercial use, distribution, and reproduction in any medium, provided the original work is properly cited. 

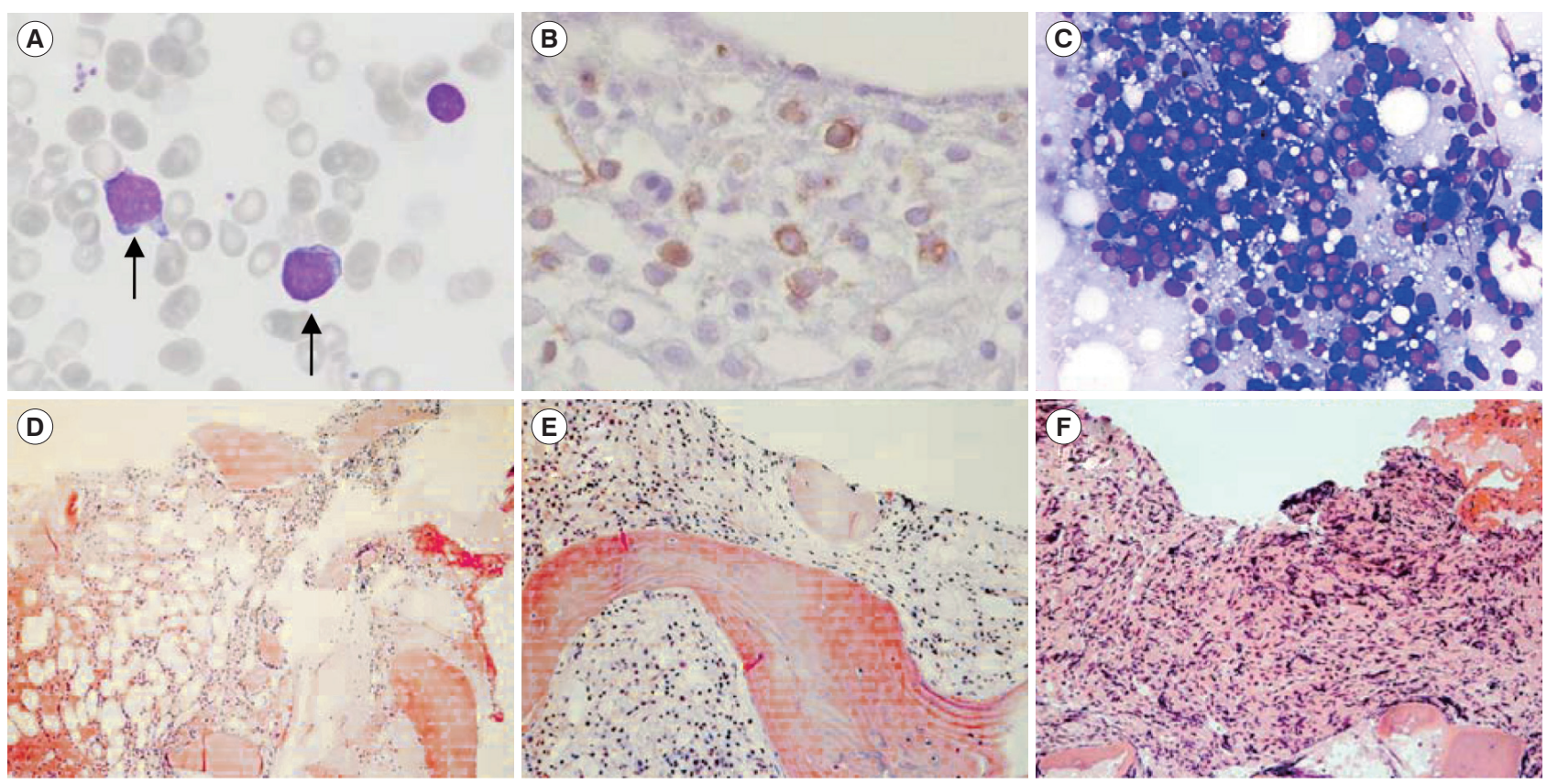

Fig. 1. Bone marrow (BM) findings. (A) Myeloblasts showing less cytoplasm and distinct prominent nucleoli at initial diagnosis (April 2011) (Wright stain, ×400); (B) CD34-positive myeloblasts in the BM biopsy (November 2011) (immunohistochemistry stain, $\times 400$ ); (C) increased immature cells in touch preparation (March 2012) (Wright stain, $\times 400$ ); (D-F) serial BM biopsies showing fibrosis progression (April 2011, November 2011, and March 2012, respectively) (hematoxylin \& eosin stain $\times 200$ ).

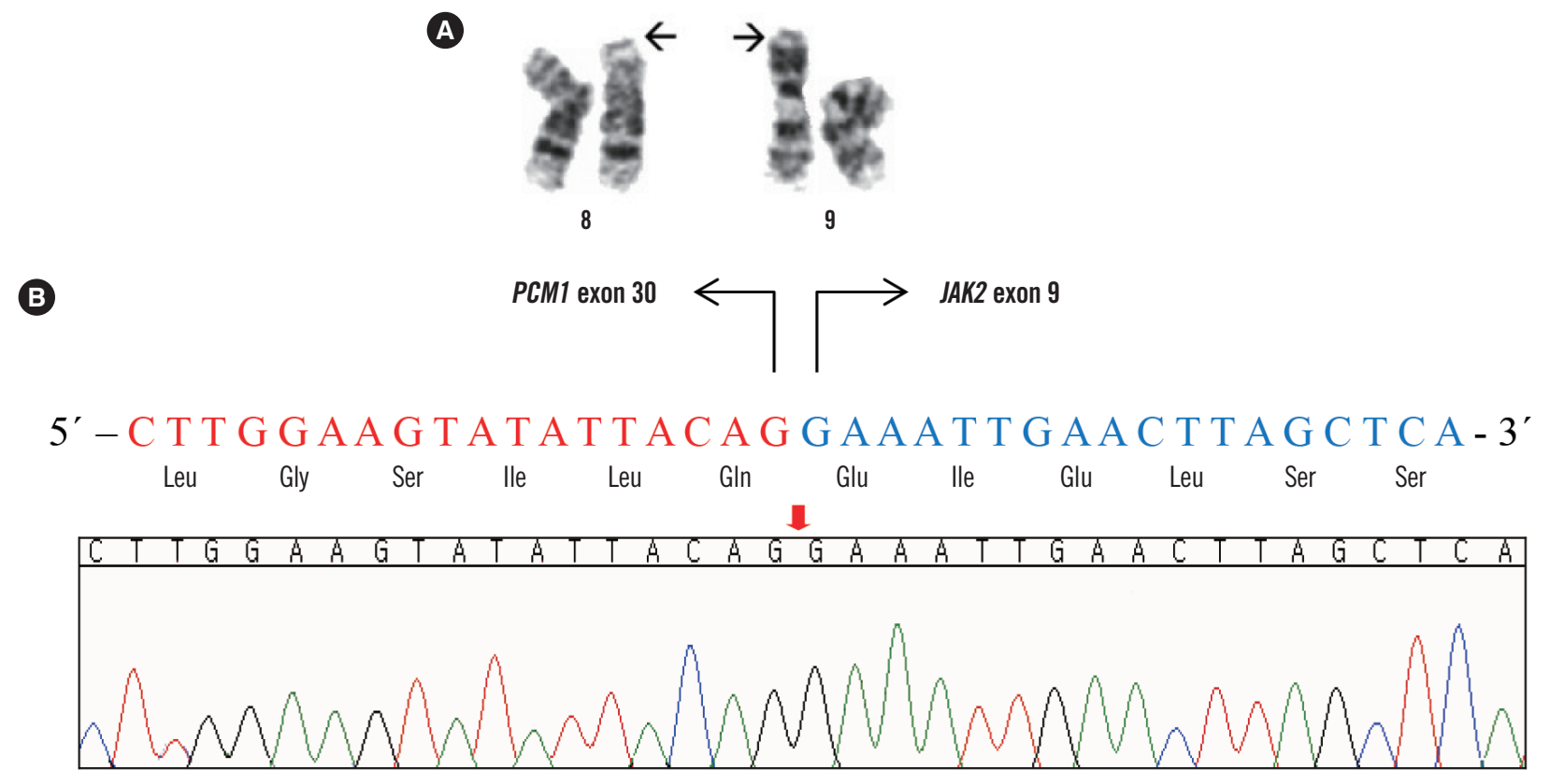

Fig. 2. The $t(8 ; 9)(p 21 ; p 24)$ translocations and $P C M 1-J A K 2$ fusion gene. (A) Karyogram of $B M$ showing $46, X X, t(8 ; 9)(p 21 ; p 24)$, (B) Sequence of the chimeric PCM1-JAK2 gene showing in-frame fusion between exon 30 of PCM1 and exon 9 of JAK2.

classification [7]. A cytogenetic examination could not be performed successfully because of inadequate specimens. The patient experienced complete remission following induction che- motherapy with an idarubicin and cytosine arabinoside regimen and subsequently received three courses of high dose consolidation therapy with cytosine arabinoside. In April 2011, the pa- 
tient re-developed pancytopenia, which lasted for three weeks; she was transferred to Seoul St. Mary's Hospital, and the BM biopsy confirmed increased myeloblasts (13\%; Fig. 1A). The patient received re-induction chemotherapy (FLANG regimen; 30 $\mathrm{mg} / \mathrm{m}^{2} /$ day fludarabine, $1 \mathrm{~g} / \mathrm{m}^{2} /$ day cytosine arabinoside, 10 $\mathrm{mg} / \mathrm{m}^{2} /$ day mitoxantrone, and $300 \mu \mathrm{g} /$ day G-CSF for five days) twice because of persistent myeloblasts in the BM. After seven months, in November 2011, the BM revealed increased myeloblasts (8\%; Fig. 1B). The patient received an allogeneic peripheral blood stem cell transplant from an HLA-matched unrelated donor. Unfortunately, her BM study revealed a relapse of acute myeloid leukemia at the 3-month follow-up post transplantation (myeloblasts 60\%, Fig. 1C). In this case, well-known phenotypes of the PCM1-JAK2 fusion, such as splenomegaly and eosinophilia, were not observed; however, myelofibrosis, a morphological feature that matches myeloid neoplasms with $P C M 1$ JAK2 was detected (Fig. 1D, 1E, and 1F). Chromosomal analyses of the specimen in November 2011, demonstrated abnormalities in the short arm of chromosome 8 and 9 (Fig. 2A): 46,XX,t(1;14)(p36.1;q11.2),t(2;6)(q35;p21.1),t(8;9)(p21;p24) [10]/46,XX[3]. We amplified the PCM1-JAK2 fusion transcript from the BM specimen with the detected chromosomal rearrangement by reverse-transcription PCR with primers designed using PRIMER 3 (available from: http://primer3.sourceforge. net): PCM1 exon 28 forward (5'-GAGCGTATGAAGACTG-3') and JAK2 exon 9 reverse (5'-GGCCATGACAGTTGCTTTGT-3'). Sanger sequencing also confirmed an in-frame fusion between PCM1 exon 30 and JAK2 exon 9 (Fig. 2B). Targeted next-generation sequencing, including 46 myeloid neoplasm-associated genes, identified a DNMT3A c.2644C > T, p.Arg882Cys mutation.

The PCM1-JAK2 fusion, together with the JAK2 V617F, MPL mutations, causes overexpression of the JAK2 pathway [5]. Therefore, a JAK2 inhibitor, ruxolitinib, could be used as a therapeutic agent; in fact, several cases with positive treatment efficacy have been reported $[8,9]$. Because eosinophilia and myelofibrosis accompany 50-70\% of myeloid neoplasms with PCM1-JAK2 [10], careful genetic evaluation is necessary for adequate diagnosis and the application of targeted therapy, even when a patient does not manifest well-known characteristics.

\section{Authors' Disclosures of Potential Conflicts of Interest}

No potential conflicts of interest relevant to this article were reported.

\section{Acknowledgments}

This study was supported by a grant from the Korea Health Technology R\&D Project, Ministry of Health and Welfare, Korea (A120175).

\section{REFERENCES}

1. Bain BJ and Ahmad S. Should myeloid and lymphoid neoplasms with PCM1-JAK2 and other rearrangements of JAK2 be recognized as specific entities? Br J Haematol 2014;166:809-17.

2. Song I, Lee DH, Lee JH, Jang S, Huh JR, Seo EJ. A t(8;9)(p22;p24)/ PCM1-JAK2 translocation in a patient with myeloproliferative neoplasm and myeloid sarcoma: first report in Korea. Ann Lab Med 2016;36:7981.

3. Arber DA, Orazi A, Hasserjian R, Thiele J, Borowitz MJ, Le Beau MM, et al. The 2016 revision to the World Health Organization classification of myeloid neoplasms and acute leukemia. Blood 2016;127:2391-405.

4. Schwaab J, Knut M, Haferlach C, Metzgeroth G, Horny HP, Chase A, et al. Limited duration of complete remission on ruxolitinib in myeloid neoplasms with PCM1-JAK2 and BCR-JAK2 fusion genes. Ann Hematol 2015;94:233-8.

5. Patterer V, Schnittger S, Kern W, Haferlach T, Haferlach C. Hematologic malignancies with PCM1-JAK2 gene fusion share characteristics with myeloid and lymphoid neoplasms with eosinophilia and abnormalities of PDGFRA, PDGFRB, and FGFR1. Ann Hematol 2013;92:759-69.

6. Reiter A, Walz C, Watmore A, Schoch C, Blau I, Schlegelberger B, et al. The $t(8 ; 9)(p 22 ; p 24)$ is a recurrent abnormality in chronic and acute leukemia that fuses PCM1 to JAK2. Cancer Res 2005;65:2662-7.

7. Arber DA, Brunning RD, Orazi A. Acute myeloid leukaemia, not otherwise specified. In: Swerdlow SH, Campo E, et al. eds. WHO classification of tumours of haematopoietic and lymphoid tissues. 4th ed. Lyon: International Agency for Research on Cancer (IARC), 2008:134-5.

8. Rumi E, Milosevic JD, Casetti I, Dambruoso I, Pietra D, Boveri E, et al. Efficacy of ruxolitinib in chronic eosinophilic leukemia associated with a PCM1-JAK2 fusion gene. J Clin Oncol 2013;31:e269-71.

9. Lierman E, Selleslag D, Smits S, Billiet J, Vandenberghe P. Ruxolitinib inhibits transforming JAK2 fusion proteins in vitro and induces complete cytogenetic remission in t (8; 9)(p22; p24)/PCM1-JAK2-positive chronic eosinophilic leukemia. Blood 2012;120:1529-31.

10. Reiter A and Gotlib J. Myeloid neoplasms with eosinophilia. Blood 2017:129:704-14. 